\title{
10 Wesentliches und Unwesentliches: Die erste Stufe der Rückfallbewegung
}

Folgerichtig beginnt das Kapitel in Teil A (GW 11, S. 245) mit dem ersten Begriffsverhältnis, das sich zwischen der Sphäre des Seins und der des Wesens nach dem einmal erfolgten Übergang und der Formulierung der generellen Aufhebung des Seins in das Wesen scheinbar natürlich einstellt. „Natürlich“ ist hier in dem Sinne gemeint, in dem Hegel in der Enzyklopädie die „erste Stellung des Gedankens zur Objektivität“ als das „unbefangne Verfahren“ (GW 20, S. 69, § 26) einer ersten, dem gewöhnlichen Verstand unmittelbar eigenen Ansicht der Verhältnisse von Natürlichem und Übernatürlichem, Teil und Ganzem, Endlichem und Absolutem, Realem und Idealem fasst. Die dort umrissene realistische, repräsentationalistische und auf den abstrakten Verstandessatz festgelegte Epistemologie setzt jedoch die Ontologie des Wesentlichen und des Unwesentlichen, des Eigentlichen und des Uneigentlichen, wie sie sich als erste Stufe der Wesenslogik erst dem Denken öffnet, bereits voraus. Denn als „Lehre von den a b stracten Bestimmungen des Wesen s “(GW 20, S. 72, § 33) von Welt, Seele und Gott und als Lehre von dem Unterschied des „wahre[n] Seyn[s]“ (GW 20, S. 74, § 36) vom bloß scheinhaften, unwahren basiert bereits die erste Stellung des Gedankens zur Objektivität, d.h. die historisch erste und entwicklungslogisch primäre Stufe des philosophischen Denkens überhaupt, auf dem Übergang von der Seins- zur Wesenslogik. ${ }^{70}$

Das Wesen ist das aufgehobene Seyn. Es ist einfache Gleichheit mit sich selbst, aber insofern es die Negation der Sphäre des Seyns überhaupt ist. So hat das Wesen die Unmittelbarkeit sich gegenüber, als eine solche, aus der es geworden ist, und die sich in diesem Aufheben aufbewahrt und erhalten hat. Das Wesen selbst ist in dieser Bestimmung seyendes, unmittelbares Wesen, und das Seyn nur ein Negatives in Beziehung auf das Wesen, nicht an und für sich selbst, das Wesen also eine bestim m te Negation. Seyn und Wesen verhalten sich auf diese Weise wieder als Andre überhaupt zueinander, denn j e d es hat ein Seyn, eine Unmittelbarkeit, die gegen einander gleichgültig sind, und stehen diesem Seyn nach in gleichem Werthe. (GW 11, S. 245)

Auf der Stufe der Unterscheidung des „Wesentliche[n]“ vom „Unwesentliche[n]“ regiert die Logik des Seins verdeckt noch beinahe vollständig die begriffliche Grammatik der Architektur des Wesens überhaupt: Folglich wird die inhaltliche

70 Daran zeigt sich, wie schwierig es ist, die verschiedenen Entwicklungsschemata bei Hegel in der Logik zu synchronisieren, da sie je verschiedene historisch-systematische Aspekte desselben einen Prozesses ausdrücken.

Ә OpenAccess. (c) 2021 Claudia Wirsing, publiziert von De Gruyter. (cc))BY Dieses Werk ist lizenziert unter einer Creative Commons Namensnennung 4.0 International Lizenz. 
Abwertung des Seins als das „Unwesentliche“ gegen das Wesen durch die logische Grammatik dieses Unterschiedes umgekehrt, die nämlich eine solche des Überdauerns seinslogischer Unterscheidung überhaupt ist. Nur dass es ab jetzt eine Dopplung von Wesen und Nicht-Wesen gibt, d. h. dass dieser Unterschied von nun an maßgeblich für jeden Zuschnitt kategorialer Differenzierungen ist, wird hier bereits unumgänglich sichtbar und kann nicht mehr negiert werden, aber wie dieser Unterschied begrifflich in Funktion gesetzt ist, und welchen Regeln er gehorcht, ist an dieser Stelle nur in einer rein äußerlichen Weise wesenslogisch gedacht: D.h. die zentralen Termini wie Wesen, Negation, Aufhebung oder Unmittelbarkeit sind hier noch bloße Worthülsen eines neuen Denkens, in welchem sich die Macht des abgelösten und verdrängten Seins als umfassende Wiederkehr manifestiert. ${ }^{71}$ Die Unmittelbarkeit des Wesentlichen gegen das Unwesentliche (das Sein) ist hier noch die seinshafte Unmittelbarkeit bloßer Sichselbstgleichheit; die negierte Unmittelbarkeit des Seins, die im Wesentlichen ,eigentlich“ aufgehoben sein soll, kehrt damit in unveränderter Form, nur äußerlich mit anderem Namen, im Wesen wieder. Die Negation als Verhältnis ist folglich wieder eine ganz äußerliche, die Sein (Unwesentliches) und Wesen wie „Andere“, d.h. gemäß der logischen Grammatik seinslogischer Äußerlichkeit, bloß wie fertige, passive Sphären auseinanderhält und nicht als lebendige innere Kraft der Selbstentwicklung bzw. Autogenerativität den gesamten Rahmen des im Wesentlichen Denkbaren ausfüllt: Somit ist nicht nur das je einzelne Relatum, sondern auch die gesamte Relation nach dem Modell seinslogischer Unterscheidung gedacht. Folglich gilt hier: „Der Unterschied von Wesentlichem und Unwesentlichem hat das Wesen in die Sphäre des Daseyn s zurückfallen lassen.“ (GW 11, S. 245) Die Frage, welche konkreten historischen Modelle des philosophischen Denkens nun alle hinter dieser eben sehr allgemeinen Typik leerer Wesensphilosophie stehen könnten - letztlich wohl alle Chorismos-Theorien von Platon bis Kant ${ }^{72}$ bzw. das platonische Paradigma von ontologischer Eigentlichkeit überhaupt ${ }^{73}$-, ist dabei weniger entscheidend als der funktionale Umstand, dass dieser Unterschied von „wesentlich“ und „unwesentlich“ keinen Unterschied macht:

71 Hegel macht das auch darin deutlich, dass für Wesen und Sein hier gilt, ihr „Anundfürsichsein“ wäre derart eine „selbst äusserliche Bestimmung“, bezüglich der „das Wesen wohl das An-und-Fürsichseyn ist, aber nur gegen Anderes, in b e st i m m ter Rücksicht“ (GW 11, S. 245). Die Einheit von Unmittelbarkeit und Bestimmtheit als innere Selbstunterscheidung des Wesens wird so zur bloß äußerlichen Kennzeichnung gegen das Andere des Seins, ohne echtes Prinzip des Wesens zu sein.

72 So Iber: Metaphysik absoluter Relationalität, S. 61.

73 Vgl. Platon: Sophistes, S. 83-89 [239b-241b]. 
Insofern daher an einem Daseyn ein Wesentliches und ein Unwesentliches von einander unterschieden werden, so ist dieser Unterschied ein äusserliches Setzen, eine das Daseyn selbst nicht berührende Absonderung eines Theils desselben, von einem anderen Theile; eine Trennung, die in ein Drittes fällt. Es ist dabey unbestimmt, was zum Wesentlichen oder Unwesentlichen gehört. Es ist irgendeine äusserliche Rücksicht und Betrachtung, die ihn macht, und derselbe Inhalt deswegen bald als wesentlich, bald als unwesentlich anzusehen. (GW 11, S. 245)

Der Unterschied des Wesentlichen und des Unwesentlichen ist aufgrund seiner seinslogischen Struktur auch in seiner Funktion rückständig, d.h. den Erfordernissen wesenslogischer Bestimmung nicht mehr angemessen: Aus ihm ergeben sich keine nicht-zufälligen, nicht-äußerlichen und nicht-relativen Normen seiner Verwendung und seines Gegenstandsbezuges. Mit anderen Worten: Wer das „Reale überhaupt“ in letztbegründender Funktion bloß in Wesentliches und Unwesentliches unterteilt, hat an und aus der Art dieser Unterteilung keinen objektiven, sachimmanenten Maßstab dafür, so und nicht anders zu unterscheiden, weil es dem Zusammenhang von Bestimmung und Bestimmtwerden, Sache und Bezugnahme objektiv so zusteht. Die umgangssprachliche starke, weil hochgradig wertnehmende Normativität der Differenz von „wesentlich“ und „unwesentlich“ ist grundlos dahingehend, nicht in einem Unterschied der Gegenständlichkeit überhaupt zu wurzeln, d.h. eine sachgegebene Unterscheidung zu sein, sondern lediglich der Sphäre eines realitätslosen Wertens anzugehören. Die noch zu weitgehende Äußerlichkeit der seinslogischen Architektur des Wesens an dieser Stelle schlägt folglich abstrakt um in die äußerlich und gegenstandslose Innerlichkeit beliebiger, subjektiver Wertsetzungen. Ex negativo wird auf diese Weise erneut die logische Notwendigkeit artikuliert, gleich in der Eröffnung der Sphäre des Wesens einen kategorialen Maßstab des „Realen überhaupt“ zu finden, der - vorgängig jeder weiteren Bestimmung - den minimalen kategorialen Zusammenhang von Äußerlichkeit und Innerlichkeit, Vorgegebensein und Setzen, Realität und Intelligibilität ein für alle Mal in eine haltbare, austarierte und vollständig funktionsfähige Begriffsform eines nicht weiter aufteilbaren und doch intern differenzierten Zusammenhangs zu übersetzen vermag. Zudem vermittelt die Erörterung des falschen Verhältnisses von „Wesentlichem“ und „Unwesentlichem“ eine ganz zentrale normative Einsicht für den weiteren Fortgang unserer besonderen Fragehinsicht in diesem Kapitel, die man pointiert so formulieren könnte: Es gibt ein falsches und ein richtiges (genetisches wie logisches) Fortleben des „Falschen“ im Wahren. Denn das überwundene, in diesem Sinne „unwahre“ weil dem logischen Entwicklungsstand des Begriffs unangemessene Denken des Seins im Ganzen soll zwar in einer bestimmten Hinsicht und für eine bestimmte logische Zeit weiterhin im Wesen als unaufhebbare Perennienz, als gegenläufiger Rest neben seiner ,korrekten` Aufhebung im Wesen, in seine 
eigene Überschreitung hineinragen - und zwar um eine gewisse normative Hinsicht von realer Äußerlichkeit im Wesen abzusichern, die für den kategorialen Minimalgrundriss des „Realen überhaupt“ notwendig ist. Doch das „wahre“ d.h. das der Stabilität und Beschreibungskraft des Logischen angemessene, Verhältnis dieses temporären Überdauerns des Seins muss erst noch gefunden werden: Hegel inszeniert auch diesen heuristischen Prozess der Findung eben jenes rechten Maßes als Mikroerzählung innerhalb der Wesenslogik über die Stufen von „wesentlich/unwesentlich“, den „Schein“ sowie die Reflexionsformen. Dabei ist es die Formulierung „,[g]enauer betrachtet“ (GW 11, S. 245), welche noch im Abschnitt zu „wesentlich/unwesentlich“ den Übergang zum „Schein“ einleitet und die der epistemologischen Funktion des pronominalen Ausdrucks ,nichts anderes als“ bei Hegel entspricht: „Der Zusatz ,nichts anderes als‘ gibt dem identifizierenden ,ist' den Sinn von ,in Wahrheit"."74 Folglich endet der Abschnitt zwischen „Wesentliche[m] und [...] Unwesentliche[m]“ damit, dass Hegel quasi resultativ erneut die darunterliegenden, „wahren“ und sich als wahre auch schon im Übergang hergestellt habenden Verhältnisse des Wesens als absolute Negativität und absolute Aufhebung des Seins bilanziert, um die Unangemessenheit des seinslogischen Unterschieds von Wesentlichem und Unwesentlichem auf dieser Stufe der Wesenslogik festzuhalten und die Rückfallbewegung des Wesens ins Sein durch die Erinnerung an dessen bereits erreichte logische Struktur zu korrigieren.

Das Wesen aber ist die absolute Negativität des Seyns; es ist das Seyn selbst, aber nicht nur als ein And er e s bestimmt, sondern das Seyn, das sich sowohl als unmittelbares Seyn, wie auch als unmittelbare Negation, als Negation, die mit einem Andersseyn behaftet ist, aufgehoben hat. Das Seyn oder Daseyn hat sich somit nicht als Anderes, denn das Wesen ist, erhalten, und das noch vom Wesen unterschiedene Unmittelbare ist nicht bloß ein unwesentliches Daseyn, sondern das an und für sich nichtige Unmittelbare; es ist nur ein Unwesen, der Schein. (GW 11, S. 245 f.)

In Wahrheit, so Hegel, sind Sein und Wesen nicht in seinslogischer Unmittelbarkeit gegeneinander gerichtete Andere, sodass sich „unmittelbares Seyn“ und „unmittelbare Negation“, d. h. vorausgegangenes Sein und die Negation des Seins im Wesen gemäß der Grammatik des „Andersseyn[s]“ verhalten. Indem das Sein bereits als das Wesen selbst in Form seiner Selbstaufhebung ins Wesen, das Wesen mithin als „die unendliche Bewegung des Seyns“ (GW 11, S. 242) selbst und kein in logischer Räumlichkeit oder Zeitlichkeit Anderes zu ihm, entwickelt worden ist,

74 Iber: Metaphysik absoluter Relationalität, S. 86. Welch große Bedeutung solche unscheinbaren grammatischen Funktionsausdrücke bei Hegel haben, führt bspw. Quante vor, wenn er die zwei Arten von Anerkennungsrelationen bei Hegel an dessen unterschiedenem Gebrauch von ,indem“ und „dadurch-dass“ entwickelt (Quante: Die Wirklichkeit des Geistes, S. 248f.). 
hat sich die Formation von „wesentlich“ und „unwesentlich“ in der Regel ihrer Unterscheidung selbst desavouiert. Die entscheidende Pointe an eben dieser Stelle ist es jedoch, dass Hegel nun - und an diesem Punkt noch uneinsehbar, aus welchen Gründen - das bereits zum zweiten Mal als vollständig aufgehoben vorgeführte Sein erneut, nun sogar in verschärfter Form, qua Umbenennung restituiert. In eben jenem (logischen) Moment, in dem durch den Vorgang der Erinnerung (,genauer betrachtet“) zum zweiten Mal die eigentlich schon erreichte Inhaltslosigkeit einer kategorialen Grammatik, die ihre Inhalte seinslogisch wie Andere und damit „Daseiende“ voneinander unterscheidet bzw. bestimmt, festgestellt wird und in dem sich folglich die daseinslogische Beziehung des Wesens zum Sein in Form von „wesentlich“ und „unwesentlich“ als vollständig inadäquat dem bereits erreichten Stand der Logik offenbart hat, wird die soeben gänzlich negierte kategoriale Matrix des Seins zum „Schein“ erklärt. Damit wird dem aufgehobenen Sein nach seiner Umbenennung zum „Unwesentlichen“ eine neuerliche Umbenennung zugemutet, die allerdings die Anfangsbedingungen und Voraussetzungen des ,Überlebens' des Seins erneut verschärft, d. h. fast bist zur Unmöglichkeit verringert: Nicht mehr das „Unwesentliche“, sondern das „Unwesen“ selbst als das „wesenlose Seyn“ (GW 11, S. 244) ist das Sein nun nur noch, d.h. sein Charakter als Verneinung des Wesens wird zugespitzt, indem er selbst sprachlich verwesentlicht wird. Des Weiteren ist das vom Wesen unabhängige, d.h. unmittelbare Sein nun nicht mehr nur bloß unwesentliches Dasein in bestimmter Beziehung zum Wesentlichen, sondern - gewissermaßen erneut in einer Verwesentlichung seines Mangels - an sich selbst und auf absolute Weise unwesentlich, d.h. „das an und für sich nichtige Unmittelbare“ (GW 11, S. 246). Damit aber erreicht die Pendelbewegung zwischen der Logik des Wesens, die das Sein vollständig als eigene Bewegung des Seins in sich aufgehoben hat und dem Rückfall in eine äußerliche Eigenständigkeit des Seins gegen das Wesen innerhalb des logischen Raums der Wesensentfaltung, ihren Höhepunkt. Bemerkenswert ist dabei die Konsequenz, mit der Hegel hier in genauer rhythmischer Komposition die seinslogische Formation im Ganzen in ihrer Geltung stufenweise entleert, bis bloß noch die ,tragische“ Auslöschung des Seins selbst, d.h. der Schein als wesentlich vernichteter, übrigbleibt. Mit der Voraussetzung eines bereits zu Beginn der Wesenslogik ungemein gedrängt entworfenen vollständigen Resultats des Übergangs des Seins in das Wesen, inklusive der Darstellung des ihm zugrunde liegenden Mechanismus der Selbstaufhebung des Seins sowie des durch den Übergang vollzogenen Umbaus kategorialer Bestimmtheit überhaupt, gewinnt Hegels Argumentation erst die normativen Mittel, um die Pendelbewegung des Übergangs stets auf das Maß des Wesens zurückzubeziehen und so die koordinierte Rückfallbewegung in den Grenzen des Fortgangs zu halten. Den erreichten Mindestgehalt an seinslogischer Reduktion jetzt aber mit dem hochgra- 
dig historisch wie normativ aufgeladenen Begriff des „Scheins“ bzw. des klassischen platonischen Gegensatzes von „Wesen“ und „Schein“ zu besetzen, eröffnet im Übergang zu den Reflexionsformen des Wesens weitere argumentative Möglichkeiten: Und es wird zu zeigen sein, wie diese Möglichkeiten letztlich für die kategoriale minimale Metastruktur von „Realität überhaupt“, wie sie sich in den Reflexionsformen inszeniert, inhaltlich notwendige Funktionen einnehmen.

Die nächsten Abschnitte meiner Argumentation werden sich damit beschäftigen, zum einen den Rückfall des Seins im Wesen herauszuarbeiten, d.h. zu zeigen, inwiefern bestimmte Geltungsansprüche des Seins (v.a. im Begriff des Scheins) im Wesen immer wieder auftreten, und zum anderen die verschiedenen Stadien dieser Bewegung offenzulegen. Dass es seinslogisch sich erhaltende Geltungsansprüche im Wesen gibt und wo diese aus welchen Gründen auftreten, wird demnach zu zeigen sein, bevor die Überführung dieser Geltungsansprüche in die reine Form der Reflexion rekonstruiert und interpretiert wird. 